\title{
Efficient Detectors based on Group Detection for Massive MIMO Systems
}

\author{
Thanh-Binh Nguyen ${ }^{1}$, Minh-Tuan Le ${ }^{2}$, Vu-Duc Ngo ${ }^{2}$, Tien-Dong Nguyen ${ }^{1}$, Huy-Dung Han ${ }^{3}$ \\ ${ }^{1}$ Le Quy Don Technical University, Hanoi, Vietnam \\ 2 MobiFone Reasearch and Development Center, MobiFone Corporation, Vietnam \\ ${ }^{3}$ Department of Electronics and Computer Engineering, Hanoi University of Science and Technology, \\ Hanoi, Vietnam
}

Correspondence: Thanh-Binh Nguyen, email: nguyenthanhbinhsqtt@gmail.com

Communication: received 15 June 2017, revised 27 August 2017, accepted 26 September 2017

Online publication: 7 March 2018, Digital Object Identifier: 10.21553/rev-jec.167

The associate editor coordinating the review of this article and recommending it for publication was Dr. Ngo Quoc Hien.

\begin{abstract}
In Multiple Input Multiple Output (MIMO) systems, the complexities of detectors depend on the size of the channel matrix. In Massive MIMO systems, detection complexity becomes remarkably higher because the dimensions of the channel matrix get much larger. In order to recover the signals in the up-link of a Massive MIMO system at reduced complexities, we first adopt the Group Detection (GD) approach to divide the system into two sub-systems. After that, we apply the Minimum Mean Square Error (MMSE) and MMSE-Bell Laboratories Layered Space-Time (MMSEBLAST) detectors to each sub-system, resulting in the so-called Group-Detection-based MMSE (MMSE-GD) and GroupDetection-based MMSE-BLAST (MMSE-BLAST-GD) detectors, respectively. To further enhance the Bit Error Rate (BER) performance of Massive MIMO systems under the high-load conditions, we propose two additional detectors, called IterativeGroup-Detection-based MMSE (MMSE-IGD) and Iterative-Group-Detection-based MMSE-BLAST (MMSE-BLAST-IGD) by respectively applying the conventional MMSE and MMSE-BLAST on the sub-systems in an iterative manner. It is shown by computer simulation and analytical results that the proposed detectors enable the system to achieve not only higher BER performance but also low detection complexities as compared to the conventional linear detectors. Moreover, the MMSE-IGD and MMSE-BLAST-IGD can significantly improve BER performance of Massive MIMO systems.
\end{abstract}

Keywords- Massive MIMO, Up-link, Low complexity receiver, Group Detection algorithm, MMSE, BLAST.

\section{INTRODUCTION}

In recent years, Massive Multiple Input Multiple Output (Massive MIMO) systems have attracted interest of many researchers because these systems can provide highly reliable communications and huge spectral/energy efficiencies [1]. In such systems, a Base Station (BS) is equipped with several hundreds of antennas to serve several tens of users (or more) in the same time-frequency resources. Basically, Massive MIMO systems are divided into two categories: Time Division Duplex (TDD) and Frequency Division Duplex (FDD). However, TDD is preferable to FDD because in a TDD system, only the number of users is limited by coherent time, whereas both the number of antennas placed at the BS and the number of users are limited in a FDD one [1].

In Massive MIMO systems, the dimensions of trans$\mathrm{mit} /$ receive signal vectors are normally very large due to large numbers of antennas and users. Hence, the optimal Maximum Likelihood (ML) detector becomes prohibitively complex and is not practical. Various conventional detectors, such as Sphere Detector (SD) or Vertical Bell Laboratory Layer Space Time (VBLAST) detectors, could also hardly be applied due to exceptionally high complexities. It was shown in [2] that
SD detector can be deployed if the number of jointly detected symbols is smaller than or equal to 32. Although the enhanced version of BLAST detector in [3] has much lower complexity as compared to that of its conventional counterpart, its computational cost is still remarkably high in Massive MIMO scenarios. Fortunately, when dimensions of the channel matrix are large enough, its columns or rows are more orthogonal in pairs. Therefore, even using linear detector such as Zero Forcing (ZF) or Minimum Mean Square Error (MMSE), the BER performance is near optimal [1]. This implies that linear detectors are good candidates for signal recovery in Massive MIMO systems. However, in such scenarios that the number of antennas is up to several hundreds (even if a thousand), the complexities of linear detectors become significant. In these situations, new detectors having lower detection complexities than those of conventional linear detectors are of necessity.

It is well-known that BER performance of a Massive MIMO system depends on the so-called load factor, $\beta$, which is defined as the ratio of the total number of transmit antennas from all users to the number of receive antenna at BS. If $\beta$ reaches $1, B E R$ performance degrades significantly. One approach of improving system performance is to utilize the traditional neighborhood search algorithms such as the Likelihood Ascent Search 
(LAS) [4], Reactive Tabu Search (RTS) [5] and multiple output selection LAS [6]. Random search (RS) and Randomized Markov chain Monte Carlo (R-MCMC) [7] or approximate message passing [8] algorithms were also proposed to achieve the goal. However, the complexities of the aforementioned detectors are still remarkably high in Massive MIMO systems.

One possible method of reducing detection complexity is to use Grouped Detection (GD), in which the size of the channel matrix is reduced before the conventional linear detectors are adopted to recover transmitted symbols. In [9], Adaptive Group Detection algorithm was firstly proposed for CDMA systems. In [10], Li et al. proposed a channel based AGD algorithm for MIMO systems. To the authors' knowledge, the idea of GD method has not been considered in Massive MIMO scenario.

In this paper, we first modify the GD approach so that it can be adopted in Massive MIMO systems. Then we propose four new low-complexity, yet efficient, detectors that are called MMSE-GD, MMSE-BLASTGD, MMSE-IGD and MMSE-BLAST-IGD. The proposed detectors are actually the combinations of the conventional linear detectors with the GD. Specifically, the MMSE-GD and MMSE-BLAST-GD are built by respectively applying MMSE and MMSE-BLAST detectors to two sub-systems generated by the GD approach. The MMSE-IGD and MMSE-BLAST-IGD detectors are constructed by applying the corresponding MMSE and MMSE-BLAST detectors to the sub-systems in an iterative manner. Simulation results demonstrate that MMSE-GD and MMSE-BLAST-GD respectively have slightly lower BER performances than those of the conventional MMSE and MMSE-BLAST detectors. In contrast, performances of the MMSE-IGD and MMSEBLAST-IGD are much higher than those of the MMSE and MMSE-BLAST, particularly when the SNR is sufficiently large. In addition, the analytical results show that compared to the MMSE detector, the MMSE-GD offers remarkably lower detection complexity, in terms of floating point operations (flops), while MMSE-IGD has slightly higher complexity. Interestingly, the complexities of both MMSE-BLAST-GD and MMSE-BLAST-IGD are much lower than that of original MMSE-BLAST one. Hence, these proposed detectors are efficient means of signal recovery in Massive MIMO systems.

The rest of the paper is organized as follows. The system model is shown in Section 2. In Section 3, we present the Group Detection approach for Massive MIMO systems and the MMSE-GD, MMSE-IGD, MMSE-BLAST-GD and MMSE-BLAST-IGD detectors. The complexity analysis and performance comparison are presented in Section 4. Finally, Section 5 concludes the paper.

\section{System Model}

In this section, we consider an up-link scenario of a single cell TDD Massive MIMO system as depicted in Figure 1. In the system, a BS is equipped with $N_{r}$

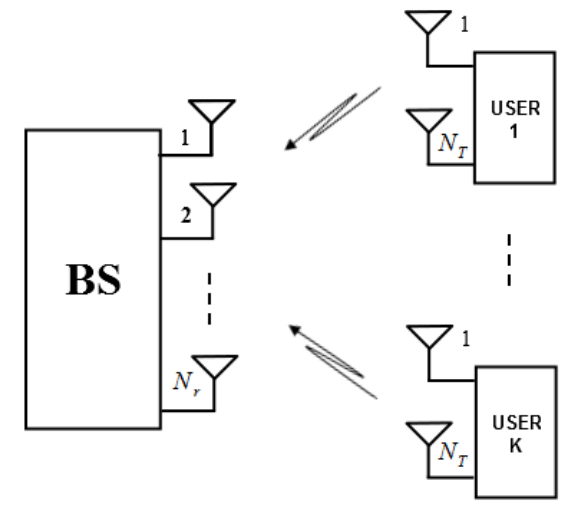

Figure 1. Up-link Massive MIMO system model.

antennas to simultaneously serve $K$ users with the same frequency resource. The number of antennas at BS and the total number of antennas deployed at user side must satisfy the condition that $N \leq N_{r}$ to avoid the problem of underdetermined system, where $N=K N_{T}$ and $N_{T}$ is the number of antennas of each user. It is assumed that each user adopts a spatial multiplexing (SMX) transmission scheme to provide high data rate. Then, the received signal at BS is the superposition of the signals transmitted from all $K$ users through wireless medium and is given by:

$$
\mathbf{y}=\sqrt{\frac{\gamma}{N E_{s}}} \sum_{k=1}^{K} \mathbf{H}_{k} \mathbf{x}_{k}+\mathbf{n}=\sqrt{\frac{\gamma}{N E_{s}}} \mathbf{H} \mathbf{x}+\mathbf{n},
$$

where $E_{S}$ is the symbol energy; $\gamma$ is the average SNR at each receive antenna; $\mathbf{y} \in \mathbb{C}^{N_{r} \times 1}, \mathbf{H} \in \mathbb{C}^{N_{r} \times N}$, $\mathbf{x} \in \mathbb{C}^{N \times 1}$ and $\mathbf{n} \in \mathbb{C}^{N_{r} \times 1}$ are the received signal vector, the channel matrix, the transmitted signal vector from $K$ users, and the noise vector, respectively; $\mathbb{C}$ is the set of complex numbers; $\mathbf{x}_{k} \in \mathbb{C}^{N_{T} \times 1}$ and $\mathbf{H}_{k} \in \mathbb{C}^{N_{r} \times N_{T}}$, $k=1, \ldots, K$, are the transmitted signal vector from the $k^{\text {th }}$ user and the channel matrix between the $k^{\text {th }}$ user and the BS. Note that $\mathbf{x}=\left[\begin{array}{llll}\mathbf{x}_{1}^{T} & \mathbf{x}_{2}^{T} & \cdots & \mathbf{x}_{K}^{T}\end{array}\right]^{T}$ and $\mathbf{H}=\left[\begin{array}{llll}\mathbf{H}_{1} & \mathbf{H}_{2} & \cdots & \mathbf{H}_{K}\end{array}\right]$. The entries of the channel matrix $\mathbf{H}$ and the noise vector $\mathbf{n}$ are assumed to be i.i.d. ${ }^{1}$ random variables with zero mean and unit variance. It is further assumed that the transmit powers from all users are equal and that transmit power from each user is divided equally among transmit antennas. This means that the covariance matrix of transmitted signal vector $\mathbf{x}$ is given by $E\left\{\mathbf{x x}^{H}\right\}=E_{s} \mathbf{I}_{N}$, where $E\{\bullet\}$ denotes expectation operation.

It is worth noting that the system model with equal power allocation among all users in (1) has been widely adopted in the context of massive MIMO, e.g, [11-15]. The most important point is that it does not alter the generality and the results of our proposed ideas. As shown below, for the same system model, our proposed detectors are capable of enhancing system performance at reduced complexities as compared to their classical counterparts. A more general system model taking into account large-scale fading effect of each user will be of our future research topic.

\footnotetext{
1 independent and identically distributed
} 
For simplicity, define $\overline{\mathbf{H}}=\sqrt{\frac{\gamma}{N E_{s}}} \mathbf{H}$, Equation (1) can be rewritten as

$$
\mathbf{y}=\overline{\mathbf{H}} \mathbf{x}+\mathbf{n} .
$$

\section{Signal Detection at Base Station}

\subsection{Group Detection Algorithm}

In this sub-section, we first modify the GD algorithm so that it can be used to enhance the reliability of signal recovery at the BS, while keeping detection complexity at reasonable levels. The details of the GD algorithm are presented as follows:

Firstly, we rewrite Equation (2) as

$$
\mathbf{y}=\left[\begin{array}{ll}
\overline{\mathbf{H}}_{1} & \overline{\mathbf{H}}_{2}
\end{array}\right]\left[\begin{array}{l}
\mathbf{s}_{1} \\
\mathbf{s}_{2}
\end{array}\right]+\mathbf{n}=\overline{\mathbf{H}}_{1} \mathbf{s}_{1}+\overline{\mathbf{H}}_{2} \mathbf{s}_{2}+\mathbf{n},
$$

where, $\overline{\mathbf{H}}_{1} \in \mathbb{C}^{N_{r} \times L}$ and $\overline{\mathbf{H}}_{2} \in \mathbb{C}^{N_{r} \times(N-L)}$ are respectively composed of the first $L$ and the remaining $(N-L)$ columns of $\overline{\mathbf{H}}$. Similarly, $\mathbf{s}_{1} \in \mathbb{C}^{L \times 1}$ and $\mathbf{s}_{2} \in \mathbb{C}^{(N-L) \times 1}$ are two sub-vectors that are created by taking the first $L$ rows and the remaining rows of $\mathbf{x}$, respectively. Generally, $L$ can take any integer value such that $1<L<N$. However, in this paper we group the channel matrices in such a way that a channel matrix corresponding to a specific user must be in one group. That is, $L=l N_{T}$, where $l$ is an integer number satisfying $1<l<K$.

Let us define $\mathbf{W}_{1}=\left(\overline{\mathbf{H}}_{1}^{H} \overline{\mathbf{H}}_{1}\right)^{-1} \overline{\mathbf{H}}_{1}^{H}$ as the pseudoinverse matrix of $\overline{\mathbf{H}}_{1}$, where $(\bullet)^{H}$ denotes Hermitian transpose operation. Then multiplying both sides of Equation (3) by $\mathbf{W}_{1}$, we obtain:

$$
\mathbf{W}_{1} \mathbf{y}=\mathbf{s}_{1}+\mathbf{W}_{1} \overline{\mathbf{H}}_{2} \mathbf{s}_{2}+\mathbf{W}_{1} \mathbf{n},
$$

or equivalently,

$$
\mathbf{s}_{1}=\mathbf{W}_{1} \mathbf{y}-\mathbf{W}_{1} \overline{\mathbf{H}}_{2} \mathbf{s}_{2}-\mathbf{W}_{1} \mathbf{n} .
$$

Substituting (5) into (3), and after some small manipulations, we get:

$$
\mathbf{y}_{2}=\tilde{\mathbf{H}}_{2} \mathbf{s}_{2}+\mathbf{n}_{2},
$$

where $\mathbf{y}_{2} \in \mathbb{C}^{N_{r} \times 1}, \tilde{\mathbf{H}}_{2} \in \mathbb{C}^{N_{r} \times(N-L)}, \mathbf{n}_{2} \in \mathbb{C}^{N_{r} \times 1}$ which are determined by $\mathbf{y}_{2}=\left(\mathbf{I}-\overline{\mathbf{H}}_{1} \mathbf{W}_{1}\right) \mathbf{y}, \tilde{\mathbf{H}}_{2}=$ $\left(\mathbf{I}-\overline{\mathbf{H}}_{1} \mathbf{W}_{1}\right) \overline{\mathbf{H}}_{2}, \mathbf{n}_{2}=\left(\mathbf{I}-\overline{\mathbf{H}}_{1} \mathbf{W}_{1}\right) \mathbf{n}$, and $\mathbf{I}$ is an $N_{r} \times$ $N_{r}$ identity matrix. It is noteworthy that the matrix $\left(\mathbf{I}-\overline{\mathbf{H}}_{1} \mathbf{W}_{1}\right)$ is singular. Hence, it is not invertible. Besides, it is straightforward to show that $E\left\{\mathbf{n}_{2}\right\}=\mathbf{0}$ and $E\left\{\mathbf{n}_{2} \mathbf{n}_{2}^{H}\right\}=\sigma^{2}\left(\mathbf{I}-\overline{\mathbf{H}}_{1} \mathbf{W}_{1}\right)^{H}$. This means that the second-order statistical characteristic of the noise vector $\mathbf{n}_{2}$ in (6) has been altered. Now, we can apply any conventional detector on sub-system (6) to obtain the estimate, $\hat{\mathbf{s}}_{2}$, of the signal vector $\mathbf{s}_{2}$. Once we obtain $\hat{\mathbf{s}}_{2}$, we assume it is the correct estimate of $\mathbf{s}_{2}$ and use it to cancel the interference effect of $\mathbf{s}_{2}$ on $\mathbf{s}_{1}$. That is, we generate the system equation to estimate $\mathbf{s}_{1}$ as follows:

$$
\mathbf{y}_{1}=\mathbf{y}-\overline{\mathbf{H}}_{2} \hat{\mathbf{s}}_{2}=\overline{\mathbf{H}}_{1} \mathbf{s}_{1}+\mathbf{n} .
$$

Again, we can apply any conventional detector on Equation (7) to obtain the estimate, $\hat{\mathbf{s}}_{1}$, of $\mathbf{s}_{1}$. For simplicity, in this paper we use the same conventional detector to recover both $\hat{\mathbf{s}}_{1}$ and $\hat{\mathbf{s}}_{2}$.

Finally, the transmitted signal vector from $K$ users, $\mathbf{x}$, is estimated by arranging $\hat{\mathbf{s}}_{1}$ and $\hat{\mathbf{s}}_{2}$ as $\hat{\mathbf{x}}=\left[\begin{array}{ll}\hat{\mathbf{s}}_{1}^{T} & \hat{\mathbf{s}}_{2}^{T}\end{array}\right]^{T}$.

\subsection{Proposed MMSE-GD and MMSE-IGD Detectors}

3.2.1 MMSE-GD: At the BS, the MMSE detector can be used to recover the transmitted vector $\mathbf{x}$ directly as

$$
\hat{\mathbf{x}}=\mathcal{Q}\left(\mathbf{W}_{m} \mathbf{y}\right),
$$

where $\mathcal{Q}(\bullet)$ is the quantization function, which estimates the transmitted signal symbols by slicing the weighted signals, $\mathbf{W}_{m} \mathbf{y}$, to the nearest values in the quantization set $\Theta=\{ \pm 1, \pm 3, \pm 5, \cdots\}$, depending on the size of the adopted QAM constellation; $\mathbf{W}_{m} \in$ $\mathbb{C}^{N \times N_{r}}$ is MMSE weight matrix and $\mathbf{W}_{m}=\left(\overline{\mathbf{H}}^{H} \overline{\mathbf{H}}+\right.$ $\left.\frac{1}{E_{\mathrm{S}}} \mathbf{I}_{N}\right)^{-1} \overline{\mathbf{H}}^{H}$. As mentioned earlier, the complexity of MMSE detector will have remarkably high complexity if it is adopted directly in Massive MIMO systems.

The proposed MMSE-GD detector recovers the transmitted signal vector by applying the conventional MMSE detector to two sub-system in (6) and (7) to obtain the estimates of $\mathbf{s}_{1}$ and $\mathbf{s}_{2}$ as follows:

$$
\begin{aligned}
& \hat{\mathbf{s}}_{1}=\mathcal{Q}\left(\mathbf{W}_{m, 1} \mathbf{y}_{1}\right), \\
& \hat{\mathbf{s}}_{2}=\mathcal{Q}\left(\mathbf{W}_{m, 2} \mathbf{y}_{2}\right),
\end{aligned}
$$

where $\mathbf{W}_{m, 1} \in \mathbb{C}^{L \times N_{r}}$ and $\mathbf{W}_{m, 2} \in \mathbb{C}^{(N-L) \times N_{r}}$ are weight matrices given by:

$$
\begin{aligned}
& \mathbf{W}_{m, 1}=\left(\overline{\mathbf{H}}_{1}^{H} \overline{\mathbf{H}}_{1}+\frac{1}{E_{s}} \mathbf{I}_{L}\right)^{-1} \overline{\mathbf{H}}_{1}^{H}, \\
& \mathbf{W}_{m, 2}=\tilde{\mathbf{H}}_{2}^{H}\left(\tilde{\mathbf{H}}_{2} \tilde{\mathbf{H}}_{2}^{H}+\frac{1}{E_{s}}\left(\mathbf{I}-\overline{\mathbf{H}}_{1} \mathbf{W}_{1}\right)^{H}\right)^{-1} .
\end{aligned}
$$

Unfortunately, the term $\left(\tilde{\mathbf{H}}_{2} \tilde{\mathbf{H}}_{2}^{H}+\frac{1}{E_{s}}\left(\mathbf{I}-\overline{\mathbf{H}}_{1} \mathbf{W}_{1}\right)^{H}\right)$ is almost singular and hence it is not invertible. In order to utilize the MMSE procedure, we approximate the weigh matrix $\mathbf{W}_{m, 2}$ as

$$
\mathbf{W}_{m, 2}=\tilde{\mathbf{H}}_{2}^{H}\left(\tilde{\mathbf{H}}_{2} \tilde{\mathbf{H}}_{2}^{H}+\frac{1}{E_{s}}\left[\left(\mathbf{I}-\overline{\mathbf{H}}_{1} \mathbf{W}_{1}\right)^{H}+\mathbf{A}\right]\right)^{-1},
$$

where $\mathbf{A} \in \mathbb{C}^{N_{r} \times N_{r}}$ denotes the additional matrix satisfying $\left[\left(\mathbf{I}-\overline{\mathbf{H}}_{1} \mathbf{W}_{1}\right)^{H}+\mathbf{A}\right]=\mathbf{I}$, then we obtain $\mathbf{W}_{m, 2}$ as follows

$$
\mathbf{W}_{m, 2}=\left(\tilde{\mathbf{H}}_{2}^{H} \tilde{\mathbf{H}}_{2}+\frac{1}{E_{s}} \mathbf{I}_{(N-L)}\right)^{-1} \tilde{\mathbf{H}}_{2}^{H} .
$$

It is worth noting that when the conventional MMSE detector is applied to the Massive MIMO system in (2), the achievable diversity order of the system is equal to $N_{r}-N+1$ [10]. By using our proposed MMSEGD detector, it is expected that the systems in (6) and (7) respectively achieve diversity orders of $N_{r}-$ $N+L+1$ and $N_{r}-L+1$. Therefore, in order for the Massive MIMO system in (2) to achieve the highest performance, the two sub-systems must attain the same diversity order. This is, $L$ should be chosen such that $N_{r}-N+L+1=N_{r}-L+1$, or equivalent $L=\frac{N}{2}$. In 
that case, the achievable diversity order of the system is expected to be $N_{r}-\frac{N}{2}+1$. Undoubtedly, the MMSEGD detector enables the system to have larger diversity, and hence higher BER performance, than the its conventional counterpart does. However, BER performance will be degraded because of approximating operation as in (14). Therefore, we expect that the achievable BER performance of the MMSE-GD is still nearly the same as that of the original MMSE one.

3.2.2 MMSE-IGD: The biggest advantage of the MMSE-GD is its lower complexity than that of the conventional MMSE detector. However, it suffers from the following disadvantages: 1 ) the noise enhancement effect due to the computation of $\mathbf{W}_{1}$; and 2) the approximation of the weight matrix in (14). As a consequence, it still results in high BERs. In order to improve the system performance, the MMSE-IGD detector is proposed in this sub-section. The idea behind the MMSE-IGD is that the transmitted signal vectors $\mathbf{s}_{1}$ and $\mathbf{s}_{2}$ in (3) should be decoded in two iterations using the MMSEGD. After each iteration, the recovered signal vectors $\hat{\mathbf{s}}_{1}$ and $\hat{\mathbf{s}}_{2}$ will be used to compute the corresponding Euclidean distance. The pair of $\left(\hat{\mathbf{s}}_{1}, \hat{\mathbf{s}}_{2}\right)$ associated with the minimum distance will be selected as the desired signal vector.

Specifically, at the first iteration, the signals are recovered using the MMSE-GD. After this iteration, we get $\hat{\mathbf{x}}=\left[\begin{array}{cc}\hat{\mathbf{s}}_{1}^{T} & \hat{\mathbf{s}}_{2}^{T}\end{array}\right]^{T}$ and compute the Euclidean distance $d_{1}$ as follows:

$$
d_{1}=\|\mathbf{y}-\overline{\mathbf{H}} \hat{\mathbf{x}}\|^{2} .
$$

At the second iteration, $\mathbf{s}_{1}$ is estimated first, followed by $\mathbf{s}_{2}$. In order to do so, we define $\mathbf{W}_{2}=\left(\overline{\mathbf{H}}_{2}^{H} \overline{\mathbf{H}}_{2}\right)^{-1} \overline{\mathbf{H}}_{2}^{H}$. By following similar steps as in the MMSE-GD, we obtain:

$$
\tilde{\mathbf{y}}_{1}=\tilde{\mathbf{H}}_{1} \mathbf{s}_{1}+\tilde{\mathbf{n}}_{1},
$$

where $\tilde{\mathbf{y}}_{1} \in \mathbb{C}^{N_{r} \times 1}, \tilde{\mathbf{H}}_{1} \in \mathbb{C}^{\left.N_{r} \times L\right)}, \tilde{\mathbf{n}}_{\mathbf{1}} \in \mathbb{C}^{N_{r} \times 1}$ which are determined by $\tilde{\mathbf{y}}_{1}=\left(\mathbf{I}-\overline{\mathbf{H}}_{2} \mathbf{W}_{2}\right) \mathbf{y}, \tilde{\mathbf{H}}_{1}=$ $\left(\mathbf{I}-\overline{\mathbf{H}}_{2} \mathbf{W}_{2}\right) \overline{\mathbf{H}}_{1}$ and $\tilde{\mathbf{n}}_{\mathbf{1}}=\left(\mathbf{I}-\overline{\mathbf{H}}_{2} \mathbf{W}_{2}\right) \mathbf{n}$. Next, we apply the conventional MMSE detector to (16) to recover $\hat{\mathbf{s}}_{1}$. After that, we use $\hat{\mathbf{s}}_{1}$ to cancel out the interference of $\mathbf{s}_{1}$ and extract $\hat{\mathbf{s}}_{2}$. Then, we get $\hat{\mathbf{x}}=\left[\begin{array}{cc}\hat{\mathbf{s}}_{1}^{T} & \hat{\mathbf{s}}_{2}^{T}\end{array}\right]^{T}$ and use it to compute the corresponding Euclidean distance $d_{2}$ as $d_{2}=\|\mathbf{y}-\overline{\mathbf{H}} \hat{\mathbf{x}}\|^{2}$. Finally, the transmitted vector, $\mathbf{x}$, is recovered by choosing the vector $\hat{\mathbf{x}}$ associated with the smallest Euclidean distance among the two distances $d_{1}$ and $d_{2}$. The MMSE-IGD detector is summarized in Algorithm 1.

\subsection{Proposed MMSE-BLAST-GD and MMSE-BLAST-IGD Detectors}

In this sub-section we propose to use the MMSEBLAST detector in the same way as to use the MMSEGD and MMSE-IGD detectors. The resulting detectors are called MMSE-BLAST-GD and MMSE-BLAST-IGD, which are described in details below.

We firstly compute the MMSE error covariance matrices $\mathbf{P}_{i}, i=1,2$, which are used to order the rows of

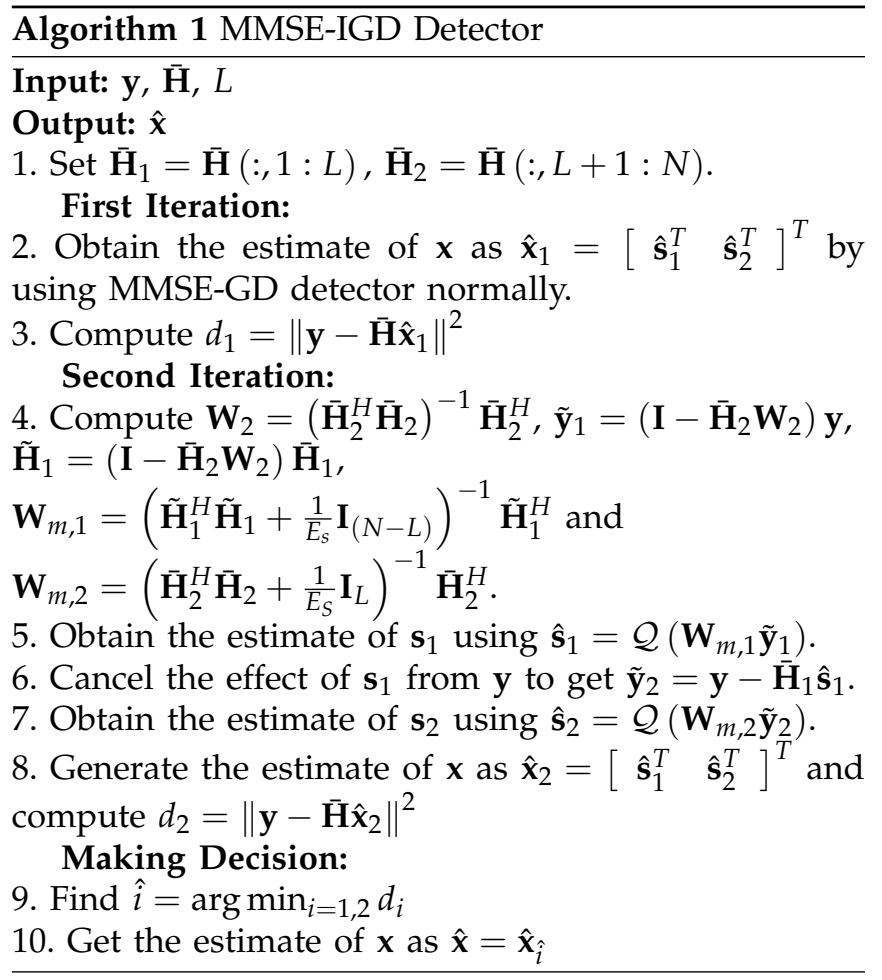

the MMSE filter matrices as follows:

$$
\begin{aligned}
& \mathbf{P}_{1}=\left(\overline{\mathbf{H}}_{1}^{H} \overline{\mathbf{H}}_{1}+\frac{1}{E_{s}} \mathbf{I}_{L}\right)^{-1}, \\
& \mathbf{P}_{2}=\left(\tilde{\mathbf{H}}_{2}^{H} \tilde{\mathbf{H}}_{2}+\frac{1}{E_{s}} \mathbf{I}_{N-L}\right)^{-1} .
\end{aligned}
$$

As already discussed in [3], the "strongest" signal among the entries of $\mathbf{s}_{i}, i=1,2$, must be the one with the smallest error covariance, i.e., the one for which $\mathbf{P}_{i}(k, k)$ (the $k^{\text {th }}$ diagonal element of $\left.\mathbf{P}_{i}\right)$ is smallest. Therefore, the diagonal entries of $\mathbf{P}_{i}$ are ordered from the biggest to smallest values, i.e., the biggest value is placed in the first diagonal entry of $\mathbf{P}_{i}$ and so on. Let $\mathbf{p}_{1} \in \mathbb{N}^{L \times 1}$ and $\mathbf{p}_{2} \in \mathbb{N}^{(N-L) \times 1}$, where $\mathbb{N}$ is the set of positive integer numbers, be the permutation vectors that define the orders of the diagonal entries of $\mathbf{P}_{1}$ and $\mathbf{P}_{2}$, respectively. In addition, let $\mathbf{G}_{1} \in \mathbb{C}^{L \times N_{r}}$ and $\mathbf{G}_{2} \in \mathbb{C}^{(N-L) \times N_{r}}$ be the ordered MMSE matrices corresponding to two sub-systems in (7) and (6). Then, we can write:

$$
\begin{aligned}
& \mathbf{G}_{1}=\mathbf{W}_{1}\left(\mathbf{p}_{1},:\right), \\
& \mathbf{G}_{2}=\mathbf{W}_{2}\left(\mathbf{p}_{2},:\right),
\end{aligned}
$$

where $\mathrm{W}_{\mathbf{1}} \in \mathbb{C}^{L \times N_{r}}$ and $\mathrm{W}_{\mathbf{2}} \in \mathbb{C}^{(N-L) \times N_{r}}$ are nonordered MMSE weight matrices for the sub-systems in (7) and (6), respectively.

By using $\mathbf{G}_{i}, i=1,2$, the $k^{\text {th }}$ entry of the recovered signal vectors, $\hat{\mathbf{s}}_{i}, i=1,2$, is obtained as follows:

$$
\hat{s}_{k, i}=\mathcal{Q}\left(\mathbf{g}_{k, i} \mathbf{y}_{i}\right),
$$

where $\mathbf{g}_{k, i}$ is the $k^{\text {th }}$ row of $\mathbf{G}_{i}$.

Under the assumption that $\hat{s}_{k, i}$ is recovered correctly, then it is used to cancel its interference on the remaining transmitted symbols as

$$
\mathbf{y}_{i}=\mathbf{y}_{i}-\mathbf{h}_{k} \hat{s}_{k, i}
$$




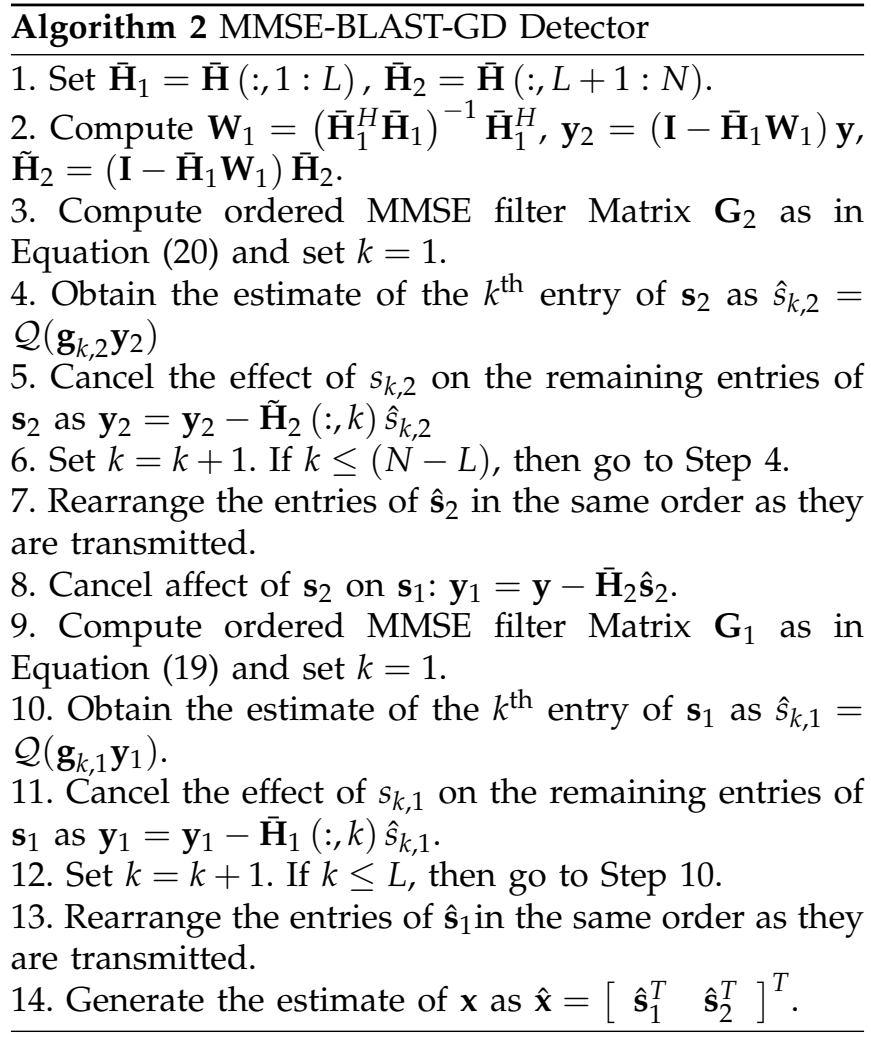

where $\mathbf{h}_{k}$ is the $k^{\text {th }}$ column of $\tilde{\mathbf{H}}_{2}$ or $\overline{\mathbf{H}}_{1}$, depending on whether $\hat{s}_{k, 2}$ or $\hat{s}_{k, 1}$ is detected. This process is repeated until all entries of $\hat{\mathbf{s}}_{1}$ and $\hat{\mathbf{s}}_{2}$ are recovered. Finally, all entries of $\hat{\mathbf{s}}_{1}$ and $\hat{\mathbf{s}}_{2}$ need to be rearranged as the same order as they are transmitted. The MMSE-BLAST-GD detector is summarized in Algorithm 2.

The MMSE-BLAST-IGD detector is constructed in the same way as the MMSE-IGD except for the fact that the MMSE-BLAST-GD detector is utilized instead of the MMSE one. The MMSE-BLAST-IGD detector is summarized in Algorithm 3.

\section{Complexity and Performance COMPARISONS}

\subsection{Complexity Comparison}

In this sub-section, we evaluate the computational complexities of MMSE-GD, MMSE-IGD, MMSEBLAST-GD and MMSE-BLAST-IGD detectors and compare them to those of the conventional MMSE and MMSE-BLAST detectors. We use the number of floating point operations (flops) to assess the complexity of these detectors. It is assumed that each real operation, e.g., either an addition, a subtraction, a multiplication, a square root, or a division, is considered a float point operation (flop). A complex addition is counted as two real operations while a complex multiplication is composed of four real multiplications and two real additions [16].

Based on the aforementioned assumptions, the complexities of the proposed detectors (i.e MMSE, BLAST, MMSE-GD, MMSE-IGD, MMSE-BLAST-GD and MMSE-BLAST-IGD) are calculated and summa-
Algorithm 3 MMSE-BLAST-IGD Detector

1. Set $\overline{\mathbf{H}}_{1}=\overline{\mathbf{H}}(:, 1: L), \overline{\mathbf{H}}_{2}=\overline{\mathbf{H}}(:, L+1: N)$.

First iteration

2. Obtain the estimate of $\mathbf{x}$ as $\hat{\mathbf{x}}_{1}=\left[\begin{array}{ll}\hat{\mathbf{s}}_{1}^{T} & \hat{\mathbf{s}}_{2}^{T}\end{array}\right]^{T}$ by using MMSE-BLAST-GD detector normally.

3. Compute $d_{1}=\left\|\mathbf{y}-\overline{\mathbf{H}} \hat{\mathbf{x}}_{1}\right\|^{2}$

\section{Second iteration}

4. Compute $\mathbf{W}_{2}=\left(\overline{\mathbf{H}}_{2}^{H} \overline{\mathbf{H}}_{2}\right)^{-1} \overline{\mathbf{H}}_{2}^{H}, \tilde{\mathbf{y}}_{1}=\left(\mathbf{I}-\overline{\mathbf{H}}_{2} \mathbf{W}_{2}\right) \mathbf{y}$, $\tilde{\mathbf{H}}_{1}=\left(\mathbf{I}-\overline{\mathbf{H}}_{2} \mathbf{W}_{2}\right) \overline{\mathbf{H}}_{1}$.

5. Compute ordered MMSE filter Matrix $\mathbf{G}_{1}$ corresponding to $\tilde{\mathbf{H}}_{1}$ as in Equation (20) and set $k=1$.

6. Obtain the estimate of the $k^{\text {th }}$ entry of $\mathbf{s}_{1}$ as $\hat{s}_{k, 1}=$ $\mathcal{Q}\left(\mathbf{g}_{k, 1} \tilde{\mathbf{y}}_{1}\right)$

7. Cancel the effect of $s_{k, 1}$ on the remaining entries of $\mathbf{s}_{1}$ as $\tilde{\mathbf{y}}_{1}=\tilde{\mathbf{y}}_{1}-\tilde{\mathbf{H}}_{1}(:, k) \hat{s}_{k, 1}$

8 . Set $k=k+1$. If $k \leq L$, then go to Step 6 .

9. Rearrange the entries of $\hat{\mathbf{s}}_{1}$ in the same order as they are transmitted.

10. Cancel affect of $\mathbf{s}_{1}$ on $\mathbf{s}_{2}: \mathbf{y}_{2}=\mathbf{y}-\overline{\mathbf{H}}_{1} \hat{\mathbf{s}}_{1}$.

11. Compute ordered MMSE filter Matrix $\mathbf{G}_{2}$ corresponding to $\overline{\mathbf{H}}_{2}$ as in Equation (20) and set $k=1$.

12. Obtain the estimate of the $k^{\text {th }}$ entry of $\mathbf{s}_{2}$ as $\hat{s}_{k, 2}=$ $\mathcal{Q}\left(\mathbf{g}_{k, 2} \mathbf{y}_{2}\right)$.

13. Cancel the effect of $s_{k, 2}$ on the remaining entries of $\mathbf{s}_{2}$ as $\mathbf{y}_{2}=\mathbf{y}_{2}-\overline{\mathbf{H}}_{2}(:, k) \hat{s}_{k, 2}$.

14. Set $k=k+1$. If $k \leq(N-L)$, then go to Step 12 .

15. Rearrange the entries of $\hat{\mathbf{s}}_{1}$ in the same order as they are transmitted.

16. Generate the estimate of $\mathbf{x}$ as $\hat{\mathbf{x}}_{2}=\left[\begin{array}{ll}\hat{\mathbf{s}}_{1}^{T} & \hat{\mathbf{s}}_{2}^{T}\end{array}\right]^{T}$ and compute $d_{2}=\left\|\mathbf{y}-\overline{\mathbf{H}} \hat{\mathbf{x}}_{2}\right\|^{2}$.

Making decision

17. Find $\hat{i}=\arg \min _{i=1,2} d_{i}$

18. Get the estimate of $\mathbf{x}$ as $\hat{\mathbf{x}}=\hat{\mathbf{x}}_{\hat{i}}$

rized as in Table I. It can be seen from Table I that the complexities of the BLAST and MMSE detectors depends only on the number of receive antennas $N_{r}$ and the total number of transmit antennas $N=K N_{T}$. Whereas, the complexities of the proposed detectors are depending on both the number of antennas and the value of $L$.

Figure 2 compares the complexities of different detectors in two antenna configurations: 1) $N_{r}=170, N=$ 160; and 2) $N_{r}=70, N=60$. In each configuration, we consider the complexities of MMSE-GD, MMSEIGD, MMSE-BLAST-GD and MMSE-BLAST-IGD detectors as a function of the variable $l$. As we can see from Figure 2, the MMSE-GD, MMSE-BLAST-GD detectors respectively have lower complexities than the conventional MMSE and MMSE-BLAST ones at almost values of $l$. We can also observe that the complexity of MMSE-BLAST-IGD detector is higher than those of all MMSE, MMSE-GD and MMSE-IGD detectors. However, it is lower than the complexity of the MMSEBLAST one when $\left\lceil\frac{1}{4} K\right\rceil<l<\left\lceil\frac{3}{4} K\right\rceil$. Interestingly, all of the MMSE-GD, MMSE-IGD, MMSE-BLAST-GD and MMSE-BLAST-IGD detectors achieve the lowest detection complexities when $l=\lceil K / 2\rceil$. Therefore, $l=$ $\lceil K / 2\rceil$ is the optimum value for the MMSE-GD, MMSE- 
Table I

COMPLEXITY COMPARISON

\begin{tabular}{|l|l|}
\hline Detector & Number of flops \\
\hline \hline MMSE & $16\left(K N_{T}\right)^{3}+32\left(K N_{T}\right)^{2} N_{r}-4\left(K N_{T}\right)^{2}-4 K N_{T} N_{r}+8\left(K N_{T}\right) N_{r}^{2}+4\left(K N_{T}\right)$ \\
\hline MMSE-BLAST & $\frac{15}{4}\left(K N_{T}\right)^{4}+2\left(K N_{T}\right)^{3} N_{r}+\left(K N_{T}\right)^{2} N_{r}^{2}+K N_{T}\left(16 N_{r}-2\right)$ \\
\hline MMSE-GD & $\begin{array}{l}16\left(K N_{T}\right)^{3}+32\left(K N_{T}\right)^{2} N r-48\left(K N_{T}\right)^{2} L+16\left(K N_{T}\right) N r^{2}+8 N r^{2}+48\left(K N_{T}\right) L^{2} \\
+48 N r L^{2}-64\left(K N_{T}\right) N r L+16 L^{2}+4\left(K N_{T}\right) N r-8 N r L-2 N r+4\end{array}$ \\
\hline \multirow{2}{*}{ MMSE-BLAST-GD } & $\frac{15}{4} L^{4}+2 L^{3} N_{r}+L^{2} N r^{2}+16 L^{3}+32 L^{2} N_{r}-4 L^{2}-12 L N_{r}+8 N_{r}^{2}-2 N_{r}+16\left(K N_{T}\right) N_{r}^{2}$ \\
& $-4\left(K N_{T}\right) N_{r}+\frac{15}{4}\left(K N_{T}-L\right)^{4}+2\left(K N_{T}-L\right)^{3} N_{r}+\left(K N_{T}-L\right)^{2} N_{r}^{2}+40 K N_{T} N_{r}-4 K N_{T}+4$ \\
\hline \multirow{2}{*}{ MMSE-IGD } & $\begin{array}{l}32\left(K N_{T}\right)^{3}+64\left(K N_{T}\right)^{2} N r-96\left(K N_{T}\right)^{2} L-4\left(K N_{T}\right)^{2}+32\left(K N_{T}\right) N r^{2}+18 N r^{2}+96 N r L^{2}+4 \\
+96\left(K N_{T}\right) L^{2}-128\left(K N_{T}\right) N r L+24\left(K N_{T}\right) N r+8\left(K N_{T}\right) L-2\left(K N_{T}\right)-2 N r-4 N r L+20 L^{2}+2 L\end{array}$ \\
\hline \multirow{3}{*}{ MMSE-BLAST-IGD } & $\begin{array}{l}16\left(K N_{T}\right)^{3}+32\left(K N_{T}\right)^{2} N_{r}-48\left(K N_{T}\right)^{2} L-4\left(K N_{T}\right)^{2}-64\left(K N_{T}\right) N_{r} L+48\left(K N_{T}\right) L^{2}+8\left(K N_{T}\right) L \\
+32\left(K N_{T}\right) N_{r}^{2}-4 N_{r}+\frac{30}{4} L^{4}+4 L^{3} N_{r}+2 L^{2} N_{r}^{2}+48 L^{2} N_{r}+8 L^{2}+6 N_{r}^{2}+\frac{30}{4}\left(K N_{T}-L\right)^{4} \\
\\
+4\left(K N_{T}-L\right)^{3} N_{r}+2\left(K N_{T}-L\right)^{2} N_{r}^{2}+80 K N_{T} N_{r}-8 K N_{T}+2\end{array}$ \\
\hline
\end{tabular}

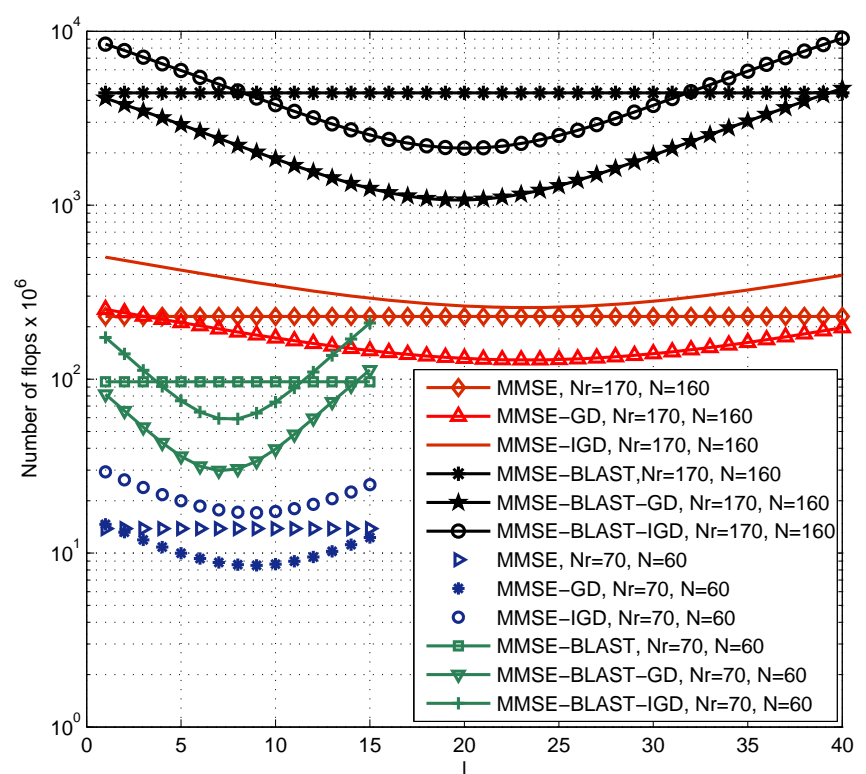

Figure 2. Complexity comparison in two configurations of antennas are $N_{r}=170, N=160$ and $N_{r}=70, N=60$ when $l$ is changed.

IGD, MMSE-BLAST-GD and MMSE-BLAST-IGD detectors regarding detection complexity.

Figure 3 represents the complexities as function of $N_{r}$ under the assumptions that $N_{r}=N, N_{T}=4$ for all users and $l$ is fixed at $\lceil K / 2\rceil$. It is clearly seen from Figure 3 that the complexity of the MMSE-GD is significantly lower than that of the MMSE while both the MMSE-BLAST-GD and MMSE-BLAST-IGD have lower complexities than the MMSE-BLAST one. On the other hand, the complexity of the MMSE-IGD is slightly higher than that of the MMSE. One can expect an increase of approximately $14.3 \%$ in complexity for sufficiently large antenna configurations. Note, however, that the higher complexity of the MMSE-IGD is compensated for by the higher BER performance as illustrated in the next sub-section. It is also seen that, there are significant gaps between MMSE-BLASTGD/MMSE-BLAST-IGD and MMSE-GD/MMSE-IGD.

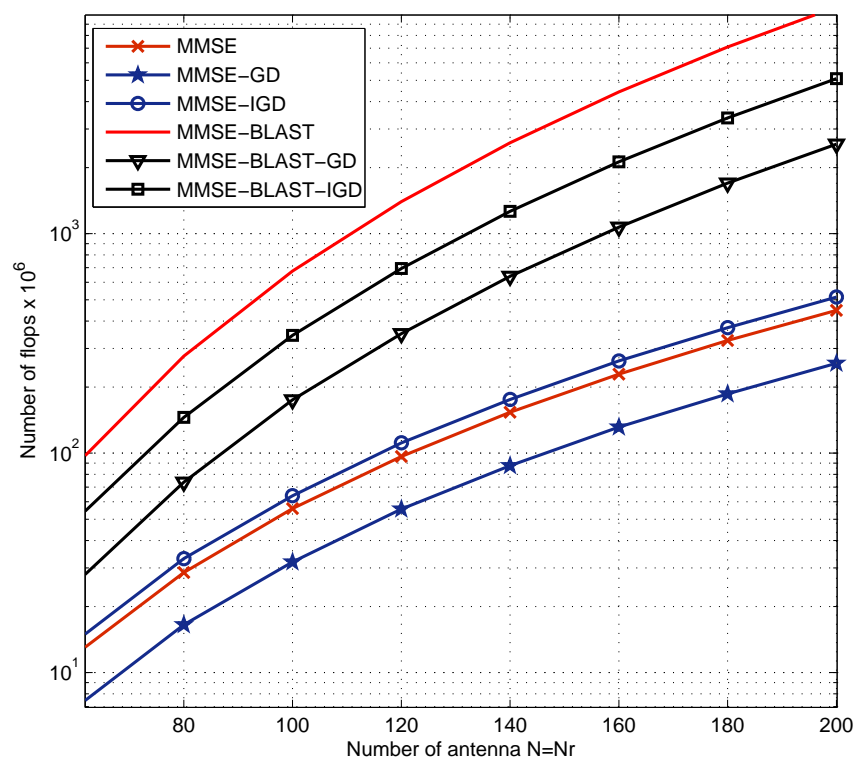

Figure 3. Detection complexities versus $N_{r}$ when $N_{T}=4 ; N_{r}=N$ and $l$ is fixed at $[K / 2\rceil$.

These gaps change when the number of antennas changes. The more antennas are equipped at BS and all users, the lager gaps are observed. This implies that the MMSE-BLAST-GD and MMSE-BLAST-IGD are suitable for systems with small or medium antennas. Whereas, MMSE-GD and MMSE-IGD detectors can be used for systems equipped with large and very large number of antennas.

\subsection{BER Performance Comparison}

In this section, the proposed detectors are compared to each others and to their conventional MMSE, MMSEBLAST counterparts with respect to BER performance. In the simulations, the channel is assumed to be quasistatic and flat Rayleigh fading. That is, the entries of the channel matrix are independent and identically distributed (i.i.d.) Gaussian random variables with zero 


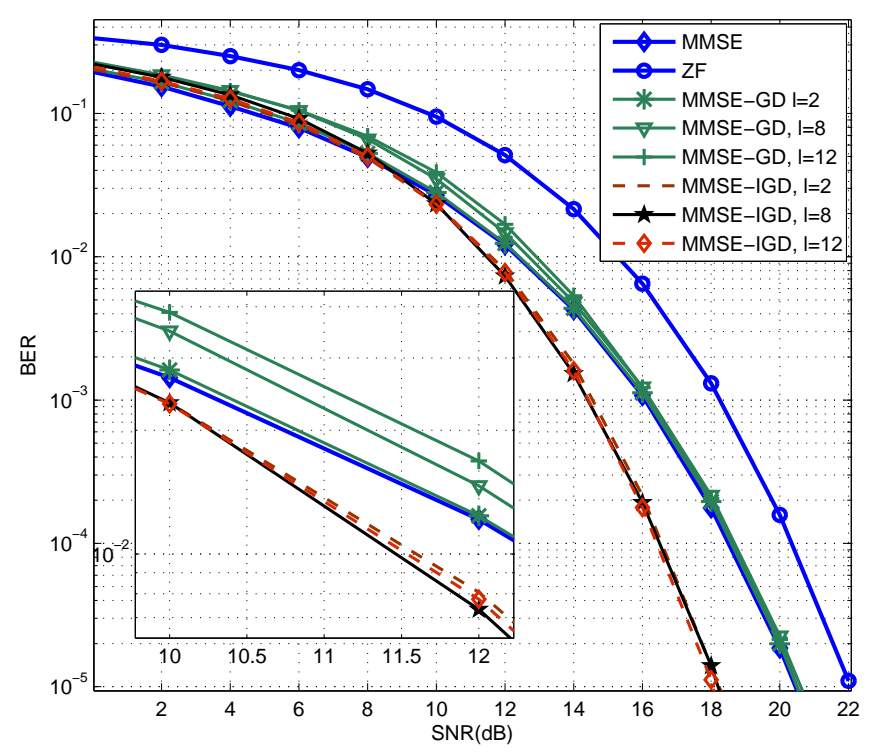

Figure 4. BER curves of the MMSE, MMSE-GD, MMSE-IGD detectors with $N_{r}=70, N=60$, 4-QAM; MMSE-GD, MMSE-IGD detectors have $l=2,8,12$.

mean and unit variance. In addition, the channel remains constant during a block of 50 symbol periods and changes independently from one block to another.

Figure 4, Figure 5, and Figure 6 represent the BER curves of MMSE-GD, MMSE-IGD, MMSE-BLAST-GD and MMSE-BLAST-IGD detectors versus those of conventional MMSE and MMSE-BLAST ones under the assumptions that $N_{r}=70, K=15, N_{T}=4$ (or equivalently $N=60$ ), and 4-QAM modulation with the average energy $E_{S}=2$. In addition, $l$ equals to 2, 8 and 12 in Figure 4 and Figure 5, whereas $l$ equals to 8 in Figure 6 . It is clearly seen from Figure 4 and Figure 5 that the MMSE-GD and MMSE-BLAST-GD have higher BERs than the corresponding MMSE and MMSE-BLAST detectors for all $l$. The BER curves of the MMSE-GD are very close to each other for different values of $l$. In other words, BER performance of the MMSE-GD is almost independent of $l$. A weak point of the MMSE-BLAST-GD detector is that its performance gets worse as $l$ increases. In contrast, the MMSE-IGD and MMSE-BLAST-IGD detectors remarkably outperform the MMSE and MMSE-BLAST ones when the SNR is sufficiently large. Specifically, at $B E R=10^{-4}$, the performance gap between the MMSE-IGD and the MMSE is about $2 \mathrm{~dB}$ with respect to the SNR. The analytical results in Figure 2 and simulation results in Figure 4 and Figure 5 implies that: 1) there is a trade-off between performance and detection complexity when the proposed detectors are used; and 2) $l$ should be set at the vicinity of $\lceil K / 2\rceil$ in order to achieve high BER performance at low detection complexity.

As mentioned earlier, a parameter that can strongly affect the BER performance of Massive MIMO systems is the load factor $\beta$. Figure 7 shows the BER curves versus $\beta$ as the ZF, MMSE, BLAST, MMSE-GD, MMSEIGD, MMSE-BLAST-GD and MMSE-BLAST-IGD detectors are applied to a Massive MIMO system with

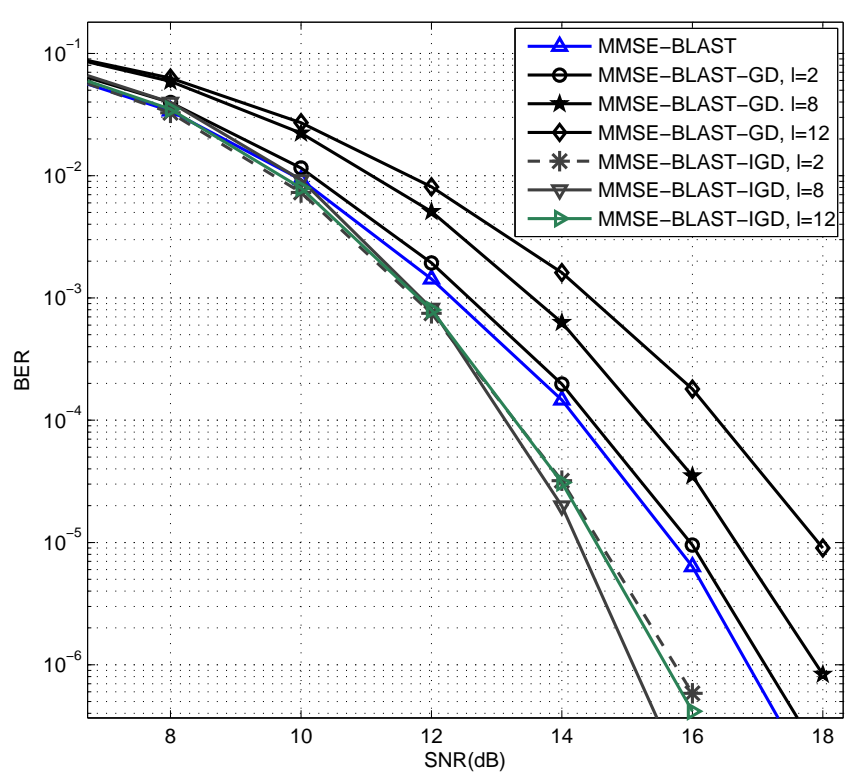

Figure 5. BER curves of the MMSE-BLAST, MMSE-BLAST-GD and MMSE-BLAST-IGD detectors with $N_{r}=70, N_{T}=4$, 4-QAM; MMSEBLAST-GD, MMSE-BLAST-IGD detectors have $l=2,8,12$.

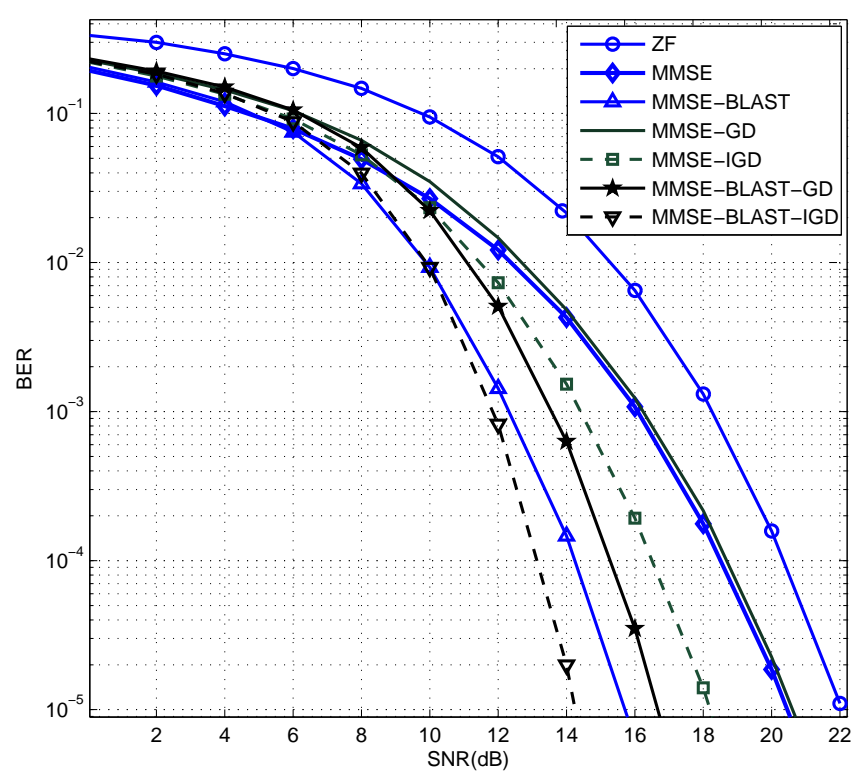

Figure 6. BER curves of the ZF, MMSE, MMSE-BLAST, MMSE-GD, MMSE-IGD, MMSE-BLAST-GD, MMSE-BLAST-IGD detectors with $N_{r}=70, N=60,4$-QAM; MMSE-GD, MMSE-IGD, MMSE-BLASTGD, MMSE-BLAST-IGD detectors have $l=\left\lceil\frac{K}{2}\right\rceil$.

$N_{r}=100$. All users use $4 Q A M$ modulation. The number of receive antenna is kept unchanged while the number of users is varied such that $\beta$ is within the range of $[0.52,1]$. The parameter $l$ is set equal to $\left\lceil\frac{K}{2}\right\rceil$ for all of the proposed detectors. As can be seen from Figure 7 , when $\beta<0.92$ the BER curve of MMSEGD very close to MMSE one. This implies that BER performance of MMSE-GD is almost the same that of MMSE. The MMSE-GD even outperforms the MMSE when $\beta<0.6$. It is also observed that the MMSE-IGD achieves a remarkable performance gap as compared 


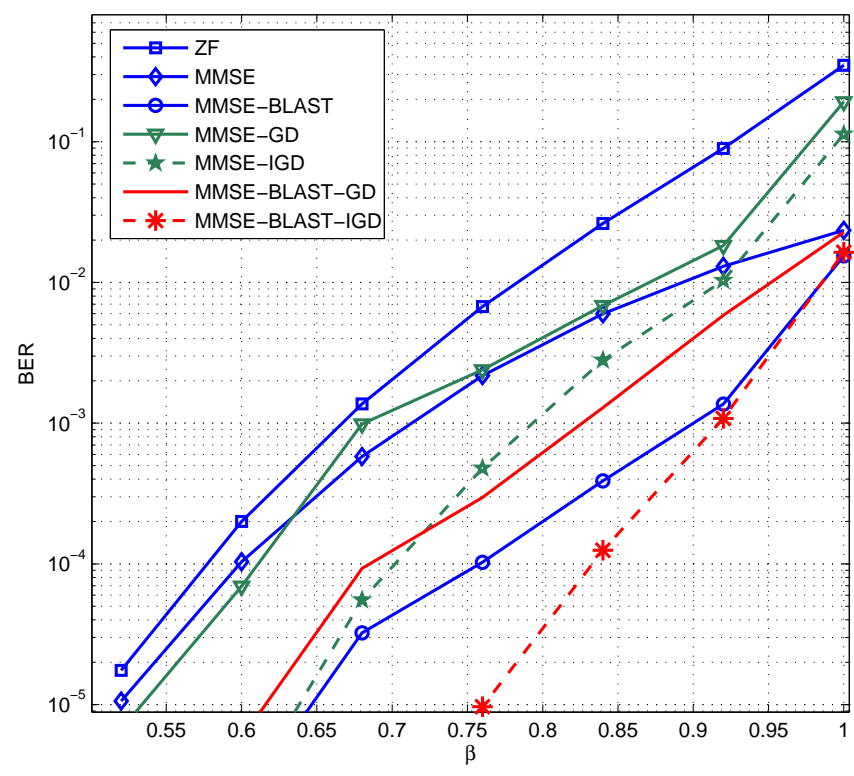

Figure 7. BER curves as functions of the load factor $\beta$ at $\mathrm{SNR}=$ $13 \mathrm{~dB}$ for the ZF, MMSE, MMSE-BLAST, MMSE-GD, MMSE-IGD, MMSE-BLAST-GD, MMSE-BLAST-IGD detectors with $N_{r}=100,4-$ QAM; MMSE-GD, MMSE-IGD, MMSE-BLAST-GD, MMSE-BLASTIGD detectors have $l=\left\lceil\frac{K}{2}\right\rceil=8$.

to MMSE one. At BER $=10^{-4}$, the MMSE-IGD enables the BS to serve approximately $103 \%, 115 \%, 117 \%$ and $121 \%$ of the loads that the MMSE-BLAST-GD, MMSEGD, MMSE and ZF detectors can handle, respectively. The Figure 7 also illustrates that the MMSE-BLAST-IGD gives the best performance for the whole range of $\beta$. If the BS uses the MMSE-BLAST-IGD, at BER $=10^{-4}$ its can server approximately $109 \%$ and $119 \%$ of the loads that the MMSE-BLAST and MMSE-IGD detectors can do.

\section{Conclusion}

In this paper, we proposed four efficient detectors based on group detection approach for Massive MIMO systems, called MMSE-GD, MMSE-IGD, MMSE-BLASTGD and MMSE-BLAST-IGD. Simulation results show that the MMSE-IGD and MMSE-BLAST-IGD detectors respectively outperforms the conventional MMSE and MMSE-BLAST ones with respect to both BER performance and computational complexity as the SNR is sufficiency high. The MMSE-GD and MMSE-BLASTGD slightly underperform the conventional MMSE and MMSE-BLAST detectors, correspondingly. Nevertheless, they offer significantly lower complexities as compared to original ones. When the BS is serving small to medium loads, using the MMSE-GD, MMSEIGD, MMSE-BLAST-GD, MMSE-BLAST-IGD is more advantageous than using the MMSE. Among the proposed detectors, the MMSE-BLAST-GD and MMSEBLAST-IGD are suitable for a small (or medium) antenna system, while the MMSE-GD and MMSE-IGD detectors can be used for the large (or very large) antenna one. Therefore, the proposed detectors are good candidate for signal recovery in the up-link of Massive MIMO systems.

\section{REFERENCES}

[1] H. Q. Ngo, Massive MIMO: Fundamentals and System Designs. Linköping University Electronic Press, 2015, vol. 1642.

[2] A. Chockalingam and B. S. Rajan, Large MIMO Systems. Cambridge University Press, 2014.

[3] B. Hassibi, "A fast square-root implementation for BLAST," in Conference Record of the Thirty-Fourth Asilomar Conference on Signals, Systems and Computers, 2000., vol. 2. IEEE, 2000, pp. 1255-1259.

[4] K. V. Vardhan, S. K. Mohammed, A. Chockalingam, and B. S. Rajan, "A low-complexity detector for large MIMO systems and multicarrier CDMA systems," IEEE Journal on Selected Areas in Communications, vol. 26, no. 3, pp. 473-485, 2008.

[5] N. Srinidhi, S. K. Mohammed, A. Chockalingam, and B. S. Rajan, "Low-complexity near-ML decoding of large non-orthogonal STBCs using reactive tabu search," in Proceedings of the IEEE International Symposium on Information Theory. IEEE, 2009, pp. 1993-1997.

[6] P. Li and R. D. Murch, "Multiple output selection-LAS algorithm in large MIMO systems," IEEE Communications Letters, vol. 14, no. 5, 2010.

[7] A. Kumar, S. Chandrasekaran, A. Chockalingam, and B. S. Rajan, "Near-optimal large-MIMO detection using randomized MCMC and randomized search algorithms," in Proceedings of the International Conference on Communications (ICC). IEEE, 2011, pp. 1-5.

[8] C. Jeon, R. Ghods, A. Maleki, and C. Studer, "Optimality of large MIMO detection via approximate message passing," in Proceedings of the International Symposium on Information Theory (ISIT). IEEE, 2015, pp. 1227-1231.

[9] M. K. Varanasi, "Group detection for synchronous Gaussian code-division multiple-access channels," IEEE Transactions on Information Theory, vol. 41, no. 4, pp. 10831096, 1995.

[10] X. Li, H. C. Huang, A. Lozano, and G. J. Foschini, "Reduced-complexity detection algorithms for systems using multi-element arrays," in Proceedings of the Global Telecommunications Conference (GLOBECOM'00), vol. 2. IEEE, 2000, pp. 1072-1076.

[11] F. Rusek, D. Persson, B. K. Lau, E. G. Larsson, T. L. Marzetta, O. Edfors, and F. Tufvesson, "Scaling up MIMO: Opportunities and challenges with very large arrays," IEEE Signal Processing Magazine, vol. 30, no. 1, pp. 40-60, 2013.

[12] H. Q. Ngo, E. G. Larsson, and T. L. Marzetta, “Energy and spectral efficiency of very large multiuser MIMO systems," IEEE Transactions on Communications, vol. 61, no. 4, pp. 1436-1449, 2013.

[13] Y. Zhang, L. Huang, J. Song, J. Li, and W. Liu, "A lowcomplexity detector for uplink massive MIMO systems based on gaussian approximate belief propagation," in Proceedings of the International Conference on Wireless Communications \& Signal Processing (WCSP). IEEE, 2015, pp. $1-5$.

[14] A. Alexandre Jr and R. Sampaio-Neto, "A random-list based LAS algorithm for near-optimal detection in largescale uplink multiuser MIMO systems," in Proceedings of the 19th International ITG Workshop on Smart Antennas (WSA 2015). VDE, 2015, pp. 1-5.

[15] X. Qin, Z. Yan, and G. He, "A near-optimal detection scheme based on joint steepest descent and jacobi method for uplink massive MIMO systems," IEEE Communications Letters, vol. 20, no. 2, pp. 276-279, 2016.

[16] T.-D. Nguyen, X.-N. Tran, T.-M. Do, V.-D. Ngo, and M.T. Le, "Low-complexity detectors for high-rate spatial modulation," in Proceedings of the International Conference on Advanced Technologies for Communications (ATC 2014). IEEE, 2014, pp. 652-656. 


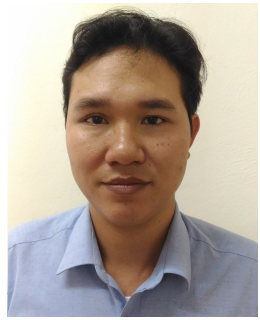

Thanh-Binh Nguyen was born in 1980 in Ha Nam, Vietnam. He received his B.E. degree in Telecommunication engineering from Telecommunication University, Vietnam in 2004, M.S. degree in Telecommunication engineering from Posts and Telecommunication Institute of Technology (PTIT), Vietnam in 2013. From 2004 to 2016 he worked as lecturer at Telecommunication university, Vietnam. He currently is working toward his Ph.D. degree nical University, Vietnam. in electronic engineering at Le Quy Don Tech-

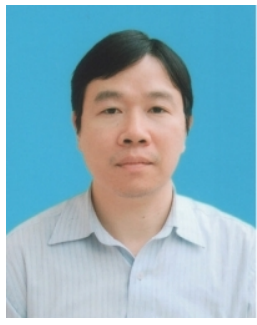

Minh-Tuan Le was born in Thanh Hoa, Vietnam, in 1976. He received his B.E. degree in electronic engineering from Hanoi University of Science and Technology, Vietnam in 1999, M.S. degree and Ph.D. degree both in electrical engineering from Information and Communication University, which is currently the Department of Electrical and Engineering of Korean Advanced Institute of Science and Technology (KAIST), Daejon, Korea, in 2003 and 2007, respectively. From 1999 to 2001 and from 2007 to 2008 he worked as a lecturer at Posts and Telecommunication Institute of Technology (PTIT), Vietnam. From November 2012 to 2015, he worked at Hanoi Department of Science and Technology, Vietnam. He is currently working at MobiFone Reasearch and Development Center, MobiFone Corporation, Vietnam. His research interests include space-time coding, space-time processing, and MIMO systems. Dr. Le is the recipient of the 2012 ATC Best Paper Award from the Radio Electronics Association of Vietnam (REV) and the IEEE Communications Society. He is a member of IEEE.

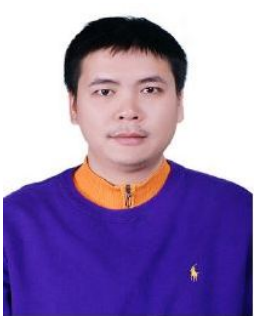

Dr. Vu-Duc Ngo is currently a lecturer at School of Electronics and Telecommunications, Hanoi University of Science and Technology, and a researcher at MobiFone Research and development Center, MobiFone corporation, Vietnam. He received the Ph.D. degree from Korea Advanced Institute of Science and Technology in 2011. During 20072009 he was a Co-founder and CTO of Wichip Technologies Inc, USA. Since 2009, he is also a Co-founder and Director of uVision Jsc, Vietnam. Since November 2012 Dr. Ngo has been serving as BoM member of the National Program on Research, Training, and Construction of High-Tech Engineering Infrastructure of Vietnam. His research interests are in the fields of $\mathrm{SoC}, \mathrm{NoC}$ design and verification, and VLSI design for multimedia codecs as well as wireless communications PHY layer. Dr. Ngo is recipient of IEEE 2006 ICCES and IEEE 2012 ATC best paper awards. Dr. Ngo is a member of IEEE.

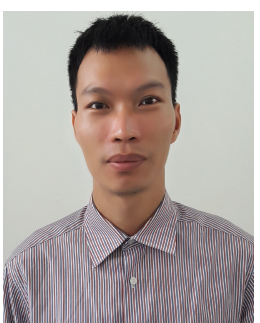

Tien-Dong Nguyen was born in Quang Tri, Vietnam in 1982. He received his B.E. and M.S. degrees in electronic engineering from Le Quy Don Technical University, Vietnam, in 2006 and 2012. From 2006 to 2010 he is a lecturer at Telecommunication University, Vietnam. He is currently working toward his $\mathrm{Ph}$.D. degree in electronic engineering at $\mathrm{Le}$ Quy Don Technical University.

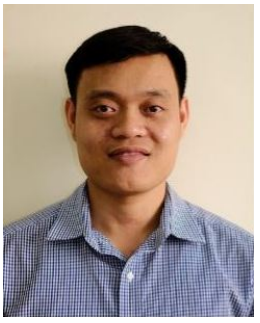

Huy-Dung Han received B.S. in 2001 from Faculty of Electronics and Telecommunications at Hanoi University of Science and Technology, Vietnam, M.Sc. degree in 2005 from Technical Faculty at University Kiel, Germany and Ph.D. degree in 2012, from the Department of Electrical and Computer Engineering at the University of California, Davis. His research interests are in the area of wireless communications and signal processing, with current emphasis on blind and semi-blind channel equalization for single and multi-carrier communication systems, convex optimization. Dr. Han is with School of Electronics and Telecommunications, Department of Electronics and Computer Engineering at Hanoi University of Science and Technology, Hanoi, Vietnam. 